Documentation et bibliothèques

\title{
La bibliothèque de l'Université du Québec à Chicoutimi : élaboration d'un mode d'organisation orienté vers le service
}

\section{Paul-Emile Boulet}

Volume 29, numéro 3, juillet-septembre 1983

URI : https://id.erudit.org/iderudit/1053622ar

DOI : https://doi.org/10.7202/1053622ar

Aller au sommaire du numéro

Éditeur(s)

Association pour l'avancement des sciences et des techniques de la documentation (ASTED)

ISSN

0315-2340 (imprimé)

2291-8949 (numérique)

Découvrir la revue

Citer cet article

Boulet, P.-E. (1983). La bibliothèque de l’Université du Québec à Chicoutimi : élaboration d'un mode d'organisation orienté vers le service. Documentation et bibliothèques, 29(3), 111-113. https://doi.org/10.7202/1053622ar

Tous droits réservés $@$ Association pour l'avancement des sciences et des techniques de la documentation (ASTED), 1983
Ce document est protégé par la loi sur le droit d'auteur. L'utilisation des services d'Érudit (y compris la reproduction) est assujettie à sa politique d'utilisation que vous pouvez consulter en ligne.

https://apropos.erudit.org/fr/usagers/politique-dutilisation/ 


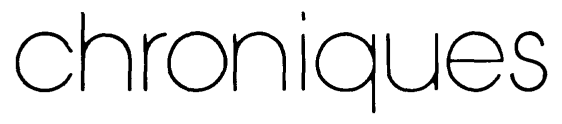

\section{La bibliothèque de l'Université du Québec à Chicoutimi: élaboration d'un mode d'organisation orienté vers le service}

Le premier juin 1982, la nouvelle bibliothèque de l'Université du Québec à Chicoutimi ouvrait ses portes.

Installée dans de nouveaux locaux et expérimentant un nouvel outil. Badaduq 11 ou SIGRID. la bibliothèque innovait de plus sur le plan de l'organisation. En effet, le déménagement coïncidait avec un réaménagement des structures internes. Ce réaménagement se voulait la concrétisation des orientations récemment approuvées'.

Pour l'essentiel, la réorganisation complétait le virage qu'avait entrepris depuis quelques années la bibliothèque de I'UOAC. Ce virage impliquait. grosso modo, un déplacement de l'objectif premier de la bibliothèque. l'accessibilité à la documentation, vers la mise à la disposition de l'information aux clients.

Ce changement de cap s'imposait de lui-même. Isolée des grands centres, la bibliothèque d'une institution de modeste envergure ne pouvait en aucun cas accéder au statut de grande bibliothèque encyclopédique susceptible d'offrir une masse innombrable et diversifiée de documents à ses usagers.

Or. sise tout de même au coeur d'une institution universitaire, la bibliothèque de I'UQAC n'en assumait pas moins la responsabilité de faire en sorte que ses chercheurs, professeurs et étudiants puissent disposer sur place des outils leur permettant de «compétionner » efficacement avec leurs confrères des meilleures institutions existantes.

Financièrement incapable de recréer sur place la bibliothèque traditionnelle de prestige, la bibliothèque de I'UQAC se devait cependant d'offrir à ses usagers le système d'information qui leur permette d'assumer pleinement leur rôle au sein d'une institution orientée vers la poursuite de l'excellence.

La collection n'était pas négligée pour autant. Cependant, on acceptait dès lors de la rationaliser en tenant compte des contraintes réelles comme

1. UQAC. Bibliothèque, Les orientations du développement de la bibliotheque de I'UOAC. Chicoutimı, 1982. la nécessité d'un fonds local minimal (besoins des étudiants du premier cycle) ou comme la perception d'un rôle d'excellence dans des secteurs particuliers liés aux axes prioritaires de l'établissement.

\section{Historique récent}

Le cadre d'organisation en place antérieurement au premier juin 1982 était, somme toute, identique dans ses grandes lignes à celui d'autres établissements semblables. II ressemblait au modèle traditionnel axé sur une division fonctionnelle entre les services au public et les services du traitement.

En pratique cependant, le modèle en question. à Chicoutimi, présentait certaines particularités. Depuis déjà six (6) ans, les professionnels des services au public intervenaient au niveau du traitement. Dès 1974. l'indexation et la classification de la documentation dans leurs disciplines particulières faisaient partie de leur mandat.

D'abord, sous la responsabilité directe du responsable du traitement, cette fonction fut confiée aux services au public en 1981.

C'est dire que, dès avant le déménagement, l'intégration des fonctions professionnelles au niveau de chaque discipline était déjà totalemet assurée. La bibliothèque de I'UQAC comptait alors dix (10) professionnels réguliers se répartissant l'ensemble des programmes développés par notre institution. Tous assumaient l'ensemble des fonctions propres à leur secteur et champ de compétence, c'est-à-dire toutes les étapes qui vont du choix du document à son exploitation, en passant par l'indexation et la classification.

Cette intégration de l'ensemble des fonctions professionnelles dans un cadre de discipline donnée n'était pas le fruit du hasard. Étant acquis que le rôle de la bibliothèque se justifiait d'abord par sa capacité de produire, au bon moment. l'information dont l'usager avait besoin, toute la logique interne dut être repensée en conséquence. 
En autres choses, la bibliothèque se devait dès lors de donner à ses diverses fonctions et activités un sens qui corresponde à l'objectif poursuivi. L'intégration des fonctions d'analyse documentaire et de classification aux activités de référence dans un domaine donné correspondait naturellement à cet objectif tout comme. d'ailleurs. l'importance prise par les programmes de formation documentaire dispensés aux usagers.

En fait, dès lors, toutes les pratiques internes. incluant les politiques de sélection du personnel. devaient être remises en question.

\section{L'étape suivante}

Le déménagement dans de nouveaux locaux fut l'occasion d'une réflexion de base sur l'opportunité de poursuivre l'expérience.

Après moult hésitations, il apparut que nous ne pouvions pas arrêter en chemin et que la responsabilité que s'était donnée la bibliothèque quant à la mise en disponibilité de l'information aux usagers ne devait pas demeurer l'exclusivité d'un groupe particulier mais devenir la responsabilité du plus grand nombre possible d'employés. Par conséquent, nous nous devions de poursuivre la marche.

Les techniciennes des services au public et du catalogage furent affectées à des secteurs précis où, associées à un ou plusieurs professionnels, elles furent appelées à assumer un éventail de fonctions élargies à l'ensemble des tâches pour lesquelles elles sont compétentes: catalogage. référence de niveau 1 , vérification et recherche bibliographique, une partie du P.E.B., etc.

Cette intégration par discipline et par niveau d'emploi devait permettre de constituer des groupes spécialisés. Ces groupes, au nombre de huit (8), constituent désormais les entités physiques de base identifiées à des groupes d'employés, à des secteurs précis, à une collection, à des équipements (terminaux ou autres) et à des locaux sis à proximité.

Les membres de chaque groupe déterminent leurs priorités et leurs objectifs en fonction de leurs perceptions des besoins des usagers.

La division des services disciplinaires est constituée de l'ensemble des groupes spécialisés par opposition à la division des services généraux chargée de la supervision de toutes les fonctions à caractère général (prêt. P.E.B. en partie, acquisition, rayonnage, reliure, etc.).

Il est de la responsabilité globale de la structure mise en place d'assurer les fonctions traditionnelles de toute bibliothèque. Ce qui caractérise notre organisation, c'est peut-être le fait que ces fonctions ne constituent plus la bàse sur laquelle s'appuie le mode d'organisation interne et que conséquemment, certaines fonctions pourront se trouver partagées entre les deux (2) divisions. C'est le cas, par exemple. du prêt entre bibliothèques.

Chacune des fonctions est supervisée par un responsable (professionnel, technicienne ou commis) appartenant à l'une ou l'autre des deux (2) divisions. Ce responsable est chargé d'animer le groupe qui lui a été confié, de définir la planification et le développement du secteur et de superviser le fonctionnement quotidien.

En pratique, le cadre d'organisation peut se résumer ainsi sous forme de tableau: 


\section{Organigramme des fonctions et identification des responsabilités de chacune des divisions Direction générale}

Services généraux

Groupe I Groupe II Groupe III Groupe IV Groupe V Groupe VI Groupe VII Groupe VII

\section{FONCTIONS}

1. Acquisitions

2. Audiovidéothèque ${ }^{1}$

3. Automatisation

4. Badaduq II (exploitation)

5. Choix

6. Comptabilité

7 Dons et échanges

8. Entretien des collections

9. Formation des usagers

10. Livres rares

11 Prêt entre biblioth.

12. Préparatıon matérielle

13. Prêt

14. Rayonnage

15. Publicité

16. Référence

17 Secrétarıat

18. Téléréférence

19. Trattement

19.1 Indexation-classification

19.2 Calalogage

Entrée des données . Fichiers SOM et TOPO

19.3 Inventaire

19.4 Repêchage

20. Vérification et recherche bibliographique

$$
\begin{gathered}
P d \\
E \\
E \\
E \\
P \\
P d \\
E \\
E \\
E \\
P
\end{gathered}
$$$$
\text { E }
$$$$
E^{\prime}
$$$$
\text { E }
$$$$
\text { E }
$$$$
\text { Pd }
$$$$
\text { E }
$$$$
P
$$$$
E^{\prime}
$$

\author{
Services disciplinaires
}

(1): Fonction confiée au Directeur

(E): Fonction exclusive à un groupe spécialisé ou aux services généraux ou à la direction générale

(P): Fonction partagée entre un groupe spécialisé, les services généraux et la direction générale

$(X d)$ : Localisation physique des personnes désignées pour superviser une fonction déterminée.

\section{Epilogue}

L'année 1982-83 fut difficile pour les membres du personnel de la bibliothèque. Tel que nous le soulignions au début, le réaménagement des structures coïncida avec le déménagement, avec l'intégration de Badaduq Il et avec les négociations collectives à l'échelle québécoise. A ceci s'est ajouté, dans le cas de nos techniciennes, un renouvellement important de notre personnel.

Après un an de travail souvent acharné. certaines des transformations prévues sont complétées, d'autres, à venir. En cours de route. des modifications ont été apportées, d'autres viendront encore.

Il est évidemment trop tôt pour évaluer l'expérience en cours ou même. peut-être pour en saisir la portée ultime.

A ce stade-ci, deux considérations s'imposent. D'une part, les membres d'une organisation, s'ils sont motivés, peuvent faire face d'emblée à certains changements. Cependant, il existe un seuil qu'on ne peut franchir sans risquer de sérieuses complications. D'autre part, il est impensable de s'engager dans une expérience semblable sans l'appui constant et le support actif de tous les membres du personnel. A Chicoutimi, nous avons pu compter sur les membres de l'organisation. Dans la plupart des occasions où des problèmes se sont posés, ce sont les personnes impliquées en première ligne qui ont envisagé elles-mêmes les solutions les plus adéquates.

Nous attendons beaucoup de la structure en place. La prochaine année devrait nous permettre de mieux cerner les possibilités réelles de l'instrument que nous nous sommes donné.

Paul-Emile Boulet, directeur

Service de la bibliothèque

Université du Québec

Chicoutimi 